\title{
THE RISE OF NEOLIBERALISM
}

\author{
Roxana STOENESCU ${ }^{\text {** }}$ \\ a) Babeș-Bolyai University, Faculty of International Relations and European \\ Studies, Cluj-Napoca, Romania
}

Please cite this article as:

Article History:

Stoenescu, R. 2019. The rise of Neoliberalism. Review of Economic Studies and Research Virgil Madgearu, 12(1), pp.73-96.

doi: 10.24193/RVM.2019.12.34.

\begin{abstract}
The current economic system with "flexible work" in the age of neoliberalism consists of accelerated production possibilities, flexible market and consumption orientation. It aims to speed up work instruction, increase the division of labor, increase flexibility and support technical achievements. How it cames to neo-liberalism, what impact it has on Western democracys and what effects it has on a post-socialist society like Romania, which had to develope within a short period of time a liberal market and become a mass consumer society will be discussed in more detail below. The main issue of this paper is to raise awareness of Romanian society problems, like the adoption, as far as possible, of the forms of Western work but without having attained the appropriate level of prosperity and living standars which leads to democartic deficits.
\end{abstract}

Key words: transitional economy; neoliberalism; planned economy; free market economy; finanical crisis; democracy deficites; population

JEL Classification: P1; P2; $P_{3} ; P_{33} ; P_{24} ; P_{25}$

(C) 2019 Alma Mater Publishing House. All rights reserved.

* Corresponding author. E-mail address: lenasvincent@yahoo.com. 


\section{References}

1. Ajder, C., Musiolek, B. and Luginbühl, C., 2014. Länderprofil Rumänien. [online] Available at <https://www.evb.ch/fileadmin/ files/documents/CCC/2014_D_CCC-CountryProfile-Romaniagerman_FINAL.pdf $>$ [Accesed on 1 July 2016].

2. Allgemeine Deutsche Zeitung, 2015. IWF: Rumänische Regierung hat Kredite zurückgezahlt. [online] Available at <http://www.adz. ro/inland/artikel-inland/artikel/iwf-rumaenische-regierung-hatkredite-zurueckgezahlt/> [Accesed on 7 June 2016].

3. Auswärtiges Amt, 2019. Rumänien - Wirtschaft. [online] Available at <https://www.auswaertiges-amt.de/de/aussenpolitik/laender/ rumaenien-node/wirtschaft/210824> [Accesed on 16 April 2019].

4. Boyer, R., 1997. French Statism and the Crossroads. In: C. Crouch and W. Streeck, ed. Political Economy of modern Capitalism. Mapping Convergence and Diversity. London: SAGE Publications, pp.71-101.

5. Brückner H.; Hauptmann A. and Vallizadeh E., 2013. IABKurzbericht. Aktuelle Analysen aus dem Institut für Arbeitsmarktund Berufsforschung: Zuwanderer aus Bulgarien und Rumänien, Arbeitsmigration oder Armutsmigration. [pdf] Available at <http:// doku.iab.de/kurzber/2013/kb1613.pdf> [Accesed on 24 June 2016].

6. Cerny, P.G., 1997. International Finance and the Erosion of Capitalist Diversity. In: C. Crouch and W. Streeck, ed. Political Economy of modern Capitalism. Mapping Convergence and Diversity. London: SAGE Publications, pp.173-82.

7. European Social Charter (EC) of 3 March 2016 on 15th National Report for the period of 01.01.2011-31.12.2014. [online] Available at $<$ https://rm.coe.int/CoERMPublicCommonSearchServices/Display DCTMContent?documentId=09000016805aagfc $>$ [Accesed on 22 June 2016].

8. Crouch, C., 2008. Postdemokratie. Frankfurt am Main: Suhrkamp Verlag.

9. Dore, R., 1997. The Distinctiveness of Japan. In: C. Crouch and W. Streeck, ed. Political Economy of modern Capitalism. Mapping Convergence and Diversity. London: SAGE Publications, pp.19-33.

10. Dimireva, I., 2012a. Starting a business in Romania. Eubusiness, [online] (last updated 13 July 2012). Available at <http://www. 
eubusiness.com/europe/romania/home/business > [Accesed on 16 April 2019].

11. Dimireva, I., 2012b. Acces to finance. Eubusiness. [online] (last updated 13 July 2012). Available at <http://www.eubusiness.com/ europe/romania/home/finance > [Accesed on April 2019].

12. Dimireva, I., 2017. Romania: Country overview, Eubusiness. [online] (last updated 30 January 2017). Available at <http://www. eubusiness.com/europe/romania> [Accesed on 16 April 2019].

13. Eubusiness, 2019. International Tax Guide Romania (Deloitte). [online] Available at <http://www.eubusiness.com/europe/ romania/business-guides-romania-deloitte/> and <https://dits. deloitte.com/\#TaxGuides $>$ [Accesed on 16 April 2019].

14. Eures (EC), 2018. Lebens- und Arbeitsbedingungen Rumänien: Einkommen und Steuer. Europäisches Portal Zur Beruflichen Mobilität. [online] Available at <https:// ec.europa.eu/eures/main.jsp?catId $=8681 \&$ acro=living\&lang= de\&parentId=7826\&countryId=RO\&living $>$ [Accesed on 18 April 2019].

15. Euroactiv, 2005. Romania introduces flat tax rate. Euroactive. [online] (last updated on o5 June 2012.) Available at <http://www. euractiv.com/section/enlargement/news/romania-introduces-flattax-rate/> [Accesed on o6 June 2016].

16. Eurokredite.de., 2018. Kredite in Rumänien. [online] (last updated 15.04 2019) Available at <http://www.eurokredite.eu/Kredite-inRumaenien/Kredite-in-Rumaenien.html> [Accesed on 27 June 2016].

17. Europäische Kommission, 2016.572 Mio. Euro für die Bekämpfung von Armut und sozialer Exklusion. [online] Available at <http:// ec.europa.eu/romania/news/15062016_572_milioane_euro_ combaterea_saraciei_excluziunii_sociale_ro.htm> [Accesed on 19 June 2016].

18. Eurostat, 2012. Agricultural census in Romania. [online] Available at <http://ec.europa.eu/eurostat/statisticsexplained/index.php/ Agricultural_census_in_Romania\#Labour_force> [Accesed on 24 June 2016].

19. Eurostat, 2013. Agricultural labour force. [online] Available at <http://ec.europa.eu/eurostat/statistics-explained/index.php/ File:Table_Agricultural_labour_force_RO_2003_and_2010. PNG $>$ [Accesed on 24 June 2016]. 
20. Eurostat, 2015a. Geburtenraten und Fruchtbarkeitsraten 200o2013. [online] Available at <http://ec.europa.eu/eurostat/ statisticsexplained/index.php/File:Live_births_and_total_ fertility_rate_in_the_2000-2013_period_in_Romania.png > [Accesed on 26 June 2016].

21. Eurostat, 2015b. Zahlen zu Eheschließungen in Rumänien 20oo2013. [online] Availableat $<$ https://ec.europa.eu/eurostat/statisticsexplained/index.php?title=File:Marriages_and_marriage_rate_ in_the_2000-2013_period_in_Romania.png > [Accesed on 23 June 2016].

22. Eurostat, 2015c. Marriages and births in Romania: Romania between modernisation and traditionalism. [online] Available at <http://ec.europa.eu/eurostat/statistics-explained/index.php/ Marriages_and_births_in_Romania > [Accesed on 26 June 2016].

23. Eurostat, 2015d. Average age at the first marriage. [online] Available at <http://ec.europa.eu/eurostat/statistics-explained/index.php/ File:Average_age_at_the_first_marriage_\%28Eurostat_data\%29. png> [Accesed on 23 June 2016].

24. Eurostat, 2019. Löhne und Arbeitskosten. [online] Available at $\quad$ https://ec.europa.eu/eurostat/statistics-explained/index. php?title=Wages_and_labour_costs/de $>$ [Accesed on 23 June 2016].

25. Fitoussi, J.P., 1997. Following the Collaps of Communism. Is there still a middle Way?. In: C. Crouch and W. Streeck, ed. Political Economy of modern Capitalism. Mapping Convergence and Diversity. London: SAGE Publications. pp.148-161.

26. Gebhardt, M., 2014. Postmoderne und Pluralität - ein demokratischer Aufbruch ins 21. Jahrhundert?. In: Donau-Institut: Working Paper Series, 36. pp.1-8. [online] Available at <http://www.andrassyuni. eu/pubfile/de-77-36-di-wp-gebhardt-final.pdf> [Accesed on 12 June 2016].

27. Gorz, A., 1967. Zur Strategie der Arbeiterbewegung im Neokapitalismus. Frankfurt am Main: Europäische Verlagsgesellschaft.

28. Graham, A., 1997. The UK 1979- 95: Myths and Realities of Conservative Cpaitalism. In: C. Crouch and W. Streeck, ed. Political Economy of modern Capitalism. Mapping Convergence and Diversity. London: SAGE Publications, pp.117-132. 
29. Group of States against Corruption (GRECO), 4 December 2015. Fourth Evaluation Round (Evaluation Report Romania), Corruption prevention in respect of members of parliament, judges and prosecutors, Strasbourg [online] Available at <http://www.coe.int/t/ $\mathrm{dghl} / \mathrm{monitoring} /$ greco/evaluations/round4/Eval\%20IV/GrecoEval 4\%282015\%294_Romania_EN.pdf> [Accesed on 7 June 2016].

30. Handelsblatt, 2007. Ungewöhnliche Praktiken in Rumänien. Kreditgeschäfte im Kaffeehaus. [online] Available at <http:// www.handelsblatt.com/unternehmen/banken-versicherungen/ ungewoehnliche-praktiken-in-rumaenien-kreditgeschaefte-imkaffeehaus-seite-2/2763878-2.html $>$ [Accesed on 27 June 2016].

31. Harvey, D., 1989. The condition of Postmodernity. USA - Cambridge Massachusetts: Blackwell.

32. Hollingsworth, J.R., 1997. The Institutional Embeddedness of American Capitalism. In: C. Crouch and W. Streeck, ed. Political Economy of modern Capitalism. Mapping Convergence and Diversity. London: SAGE Publications, pp.133-147.

33. Human Development Report, 2015. Briefing note for countries on the 2015 Human Development Report Romania. [pdf], Available at <http://hdr.undp.org/sites/all/themes/hdr_theme/country-notes/ ROU.pdf $>$ [Accesed on 23 June 2016].

34. Kerr, C., Harbison, F.H. and Myers, C.A., 1960. Industrialism and Industrial Man: The Problems of Labor and Management in Economic Growth. Cambridge, MA: Harvard University Press.

35. Konzett, E., 2016. Banken droht in Rumänien Ungemach. Wirtschaftsblatt. [online] Available at <http://wirtschaftsblatt. at/home/nachrichten/europa_cee/4966132/Banken-droht-inRumaenien-Ungemach $>$ [Accesed on 27 June 2016].

36. Laclau, E. and Mouffe C., 2001. Hegemony and Socialist Strategy. Towards a Radical Democratic Politics. London: Verso.

37. Lescu, C. and Mihalcescu, D., 2015. EU-Bildungsbericht 2015: Große Chancenungleichheit im rumänischen Schulsystem. Radio Romania International: Sozialreport - der rumänische Alltag. [online] Available at <http://www.rri.ro/de_de/ eu_bildungsbericht_2015_grosse_chancenungleichheit_im_ rumaenischen_schulsystem-2541281> [Accesed on 18 June 2016].

38. Marchart, O., 2008. Äquivalenz und Autonomie. Vorbemerkungen zu Ch. Mouffes Demokratietheorie. In: Ch. Mouffe, ed. Das demokratische Paradox. Wien: Verlag Turia + Kant. pp.7-15. 
39. Mouffe, C., 2008. Das demokratische Paradox. Wien: Verlag Turia + Kant.

40. Mouffe, C., 1979. Gramsci and Marxis Theory. Gramsci and Hegemony. London-Boston-Henley: Routledge \& Kegan Paul.

41. Naumescu, V., 2015. Experimentul Frankenstein bântuie Europa: explozia populismelor și sfârşitul ideii europene. 22 Plus, no. 355.

42. Macours, K. and Swinnen, J.F.M., 2007. Rural Poverty in Transition Countrys. LICOS-Centre for Institutions and Economic Performance: Discussion papers 169/2007, Katholieke Universiteit Leuven, [pdf], Available at <http://feb.kuleuven.be/drc/licos/ publications/dp/dp169.pdf $>$ [Accesed on 23 June 2016].

43. OECD, 2012. Pisa - Internationale Schulleistungsstudie der OECD, [online] Available at <http://www.oecd.org/berlin/themen/pisainternationaleschulleistungsstudiederoecd.htm $>, \quad<$ http://www. oecd.org/berlin/themen/PISA-2012-Zusammenfassung.pdf> and <http://www.oecd.org/pisa/aboutpisa/romania-pisa.htm> [Accesed on 1 July 2016].

44. Plehwe, D., 2009. Introduction. In: Ph. Mirowski and D. Plehwe, ed. The Road from Mont Pelerin: The Making of the Neoliberal Thought Collective. Cambridge: Harvard University Press.

45. Reinhard, W., 2006. Lebensformen Europas. Eine historische Kulturanthropologie. München: C.H. Beck.

46. Roconnet, 2017. Löhne und Gehälter / Sozialabgaben Rumänien. [online] Available at <http://roconnect.eu/infos-rumanien/lohnegehalter-sozialabgaben-rumaenien/> [Accesed on 20 March 2019].

47. Ruggie, J.G., 1998. Globalization and the Embedded Liberalism Compromise: The End of an Era?. In: W. Streeck, ed. Internationale Wirtschaft, Nationale Demokratie. Herausforderungen für die Demokratietheorie. Frankfurt am Main/New York: Campus Verlag. pp.79-99.

48. Sennet, R., 2008. Der flexible Mensch. Berlin: Berliner Taschenbuch Verlag.

49. Stiglitz, J., 2011. Im freien Fall. Vom Versagen der Märkte zur Neuordnung der Weltwirtschaft. München: Pantheon.

50. CEIC Data, 2018. Rumänien Wachstum reales BIP. [online] Available at <https://www.ceicdata.com/de/indicator/romania/ real-gdp-growth $>$ [Accesed on 18 April 2019].

51. Streeck, W., 1997. German Capitalism: Does it Exist? Can it survive?. In: C. Crouch and W. Streeck, ed. Political Economy of modern 
Capitalism. Mapping Convergence and Diversity. London: SAGE Publications, pp. 33-54.

52. Streeck, W., 2009. Re-forming Capitalism, Institutional Change in the German Political Economy. Oxford: Oxford University Press.

53. Streeck, W., 2013. Gekaufte Zeit. Die vertagte Krise des demokratischen Kapitalismus. Bonn: Bundeszentrale für politische Bildung. Berlin: Suhrkamp Verlag.

54. Statista: Das Statistik- Portal, 2016. Gesetzliche Mindestlöhne pro Stunde in Ländern der Europäischen Union. [online] Available at <http://de.statista.com/statistik/daten/studie/37401/umfrage/ gesetzliche-mindestloehne-in-der-eu/> [Accesed on 1 July 2016].

55. TAZ (Die Tageszeitung) 2004. Rumänien nimmt Kurs auf EU. Taz Archiv. [online] (Last modified on 30 December), Available at $<$ http://www.taz.de/1/archiv/?dig=2004/12/30/a0090 > [Accesed on 19 June 2016].

56. Ther, P., 2014. Die neue Ordnung auf dem alten Kontinent. Eine Geschichte des neoliberalen Europa. Frankfurt am Main: Suhrkamp Verlag.

57. Topagrar online, 2010. Rumänien: Viel Land und wenig Geld, [online] Available at <http://www.topagrar.com/archiv/ Rumaenien-Viel-Land-und-wenig-Geld-561814.html> [Accesed on 27 June 2016].

58. Trading Economics, 2019. Romania Personal income tax rate, [online] Available at <https://tradingeconomics.com/romania/ personal-income-tax-rate $>$ [Accesed on 17 April 2019].

59. Vogel, J., 2013. Transnationale Familien Rumäniens: Wie Kinder und Jugendliche die Arbeitsmigration ihrer Eltern miterleben. Hamburg: Diplomica Verlag.

6o. Păun, C., 2018. Lieber für einen Hungerlohn nach Deutschland als ein Leben in Rumänien. Welt-Wirtschaft. [online] Available at <https://www.welt.de/wirtschaft/article180815218/RumaenienDie-einzige-Zukunft-des-Landes-ist-das-Ausland.html> [Accesed on 20 March 2019].

61. The World Bank, 2018. Romania Systematic Country Diagnostic. [pdf], Available at <http://documents.worldbank.org/curated/ en/210481530907970911/pdf/128064-SCD-PUBLIC-P160439RomaniaSCDBackgroundNoteMigration.pdf> [Accesed on 20 March 2019]. 
62. Wirtschaftskammer Österreich, 2019. Öffentliche Verschuldung: Staatsschuldenquote. [online] Available at <http://wko.at/statistik/ eu/europa-verschuldung.pdf $>$ [Accesed on 16 April 2019]. 